03.1;01.1

\title{
Рост плотности и расхода тяжелого компонента при истечении разреженной газовой смеси в вакуум
}

\author{
(C) Н.Ю. Быков ${ }^{1}$, В.В. Захаров ${ }^{2}$ \\ ${ }^{1}$ Санкт-Петербургский политехнический университет Петра Великого, Санкт-Петербург, Россия \\ ${ }^{2}$ Istituto di Astrofisica e Planetologia Spaziali, Rome, Italy \\ E-mail: nbykov2006@yandex.ru
}

Поступило в Редакцию 2 апреля 2020г.

В окончательной редакции 2 апреля 2020г.

Принято к публикации 13 апреля 2020г.

\begin{abstract}
Проведено численное исследование газодинамики процессов истечения разреженной бинарной газовой смеси с большим отношением масс компонентов из источника в вакуум. Расчеты выполнены методом прямого статистического моделирования Монте-Карло. Установлен эффект роста плотности тяжелого компонента в окрестности выходного отверстия источника. Показано существенное влияние легкого компонента на газодинамику смеси, приводящее к увеличению расхода и усилению эффекта осевой фокусировки тяжелого компонента.
\end{abstract}

Ключевые слова: динамика разреженного газа, бинарная газовая смесь, истечение в вакуум, расход газа, газоструйные технологии.

DOI: 10.21883/PJTF.2020.15.49738.18328

Процессы истечения газовой смеси с большой разницей масс компонентов широко используются в различных технических приложениях. Среди последних можно указать технологии напыления наноструктурных пленок и синтеза нанокластеров в индустрии наносистем [1,2]. Например, технологии вакуумного газоструйного осаждения (ВГСО) кластерных покрытий благородных металлов предполагают истечение паров серебра (золота) из тигля совместно с несущим газом гелием. В этом случае отношение масс компонентов металл/гелий составляет 26.95 для серебра и 49.24 для золота. Для метода ВГСО характерны малые давления торможения смеси в источнике [3]. Истечение в вакуум происходит, как правило, в переходном по числу Кнудсена режиме $(0.001<\mathrm{Kn}=\lambda / D<1, \lambda$ - длина свободного пробега частиц в смеси, $D$ - диаметр выходного отверстия), и можно ожидать проявления эффектов, связанных с относительно небольшим числом взаимных столкновений частиц легкого и тяжелого компонентов.

Исследованию течений разреженного газа через отверстия или каналы малой длины при большом перепаде давлений в сообщающихся резервуарах посвящено большое число работ, однако проведенные исследования касались случая течения однокомпонентного газа или воздуха с малым отношением масс основных компонентов. Настоящая работа посвящена исследованию истечения разреженной бинарной смеси серебро-гелий из источника в вакуум через отверстие в бесконечно тонкой стенке.

Расчеты выполнены методом прямого статистического моделирования Монте-Карло [4]. Столкновения частиц разыгрывались согласно модели твердых сфер с диаметрами $d_{1}=2.9 \cdot 10^{-10} \mathrm{~m}, d_{2}=2.19 \cdot 10^{-10} \mathrm{~m}$, $d_{12}=\left(d_{1}+d_{2}\right) / 2\left(d_{1}, d_{2}, d_{12}\right.$ - диаметры столкновений класса серебро-серебро, гелий-гелий и серебро-гелий соответственно) [5,6]. Здесь и далее индекс 1 относится к серебру, 2 - к гелию, индекс 0 обозначает условия в источнике. Температура торможения смеси в источнике принята равной $T_{0}=2100 \mathrm{~K}$, радиус выходного отверстия $R=1.5 \mathrm{~mm}$. Плотность торможения $n_{0}=p_{0} k T_{0}$ $\left(p_{0}\right.$ - давление торможения, $k-$ постоянная Больцмана), мольная доля серебра $X_{0,1}$ и число Кнудсена для смеси $\mathrm{Kn}=\lambda_{0} /(2 R) \quad\left(\lambda_{0}-\right.$ длина свободного пробега частиц в источнике) для рассматриваемых вариантов приведены в таблице. Расчеты выполнены с использованием кода СПбПУ [7].

Проведенное исследование позволило установить эффект роста плотности $n_{1}$ тяжелого компонента (атомов серебра) в области выходного отверстия источника. Эффект демонстрируется с помощью данных, представленных на рис. 1, a. В случае свободномолекулярного $(\mathrm{Kn} \rightarrow \infty)$ или континуального $(\mathrm{Kn} \rightarrow 0)$ истечения смеси, а также в случае истечения однокомпонентного газа, зависимость плотности от расстояния представляет собой монотонно убывающую функцию с максимумом $n_{1} / n_{0,1}=1$ внутри источника и минимумом $n_{1} / n_{0,1}=0$

Параметры расчета

\begin{tabular}{c|c|c|c}
\hline Вариант & $n_{0}, \mathrm{~m}^{-3}$ & $X_{0,1}$ & $\mathrm{Kn}$ \\
\hline 1 & $4.5 \cdot 10^{20}$ & 0.25 & 2.83 \\
2 & $4.5 \cdot 10^{23}$ & 0.25 & $2.83 \cdot 10^{-3}$ \\
3 & $0.25 \cdot\left(4.5 \cdot 10^{22}\right)$ & 1 & $7.93 \cdot 10^{-2}$ \\
4 & $4.5 \cdot 10^{22}$ & 0.25 & $2.83 \cdot 10^{-2}$ \\
5 & $4.5 \cdot 10^{22}$ & 0.15 & $3.08 \cdot 10^{-2}$ \\
6 & $4.5 \cdot 10^{22}$ & 0.05 & $3.34 \cdot 10^{-2}$
\end{tabular}



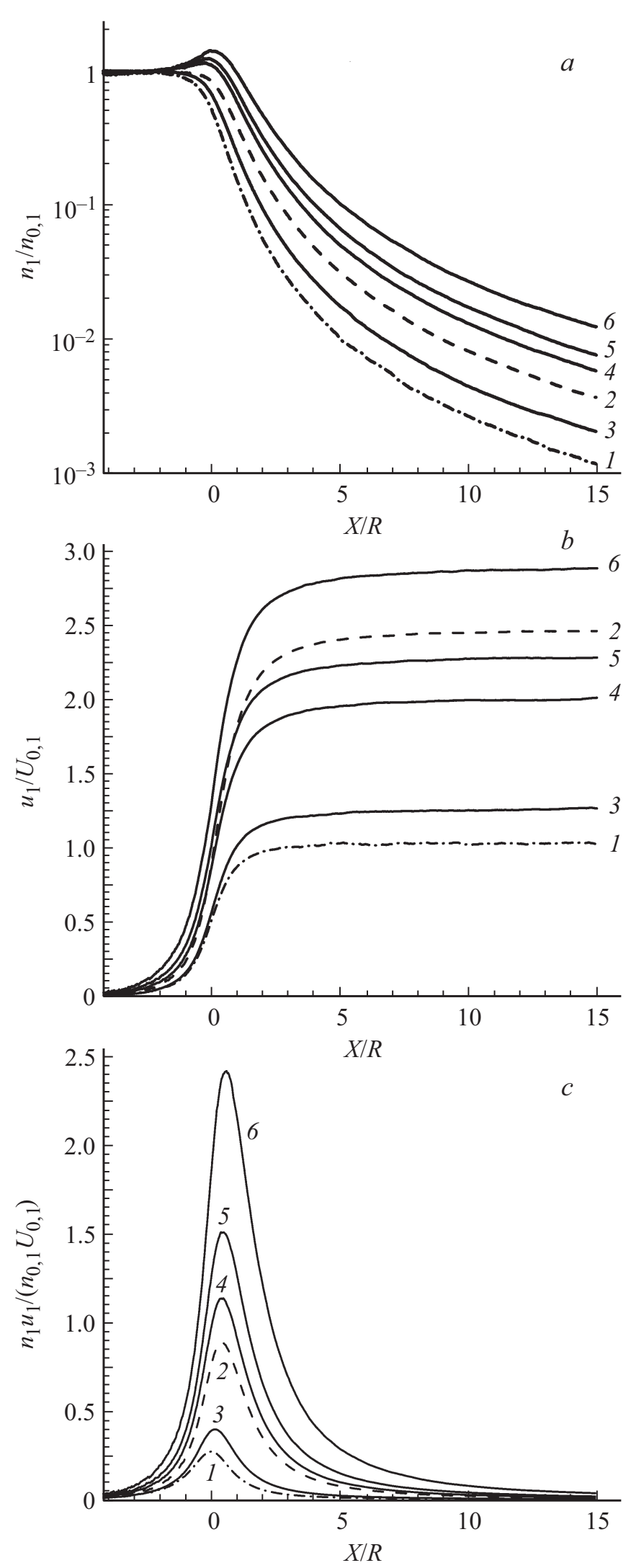

Рис. 1. Распределение плотности $(a)$, скорости $(b)$ и расхода серебра $(c)$ вдоль оси течения. Номера кривых соответствуют номерам вариантов в таблице.

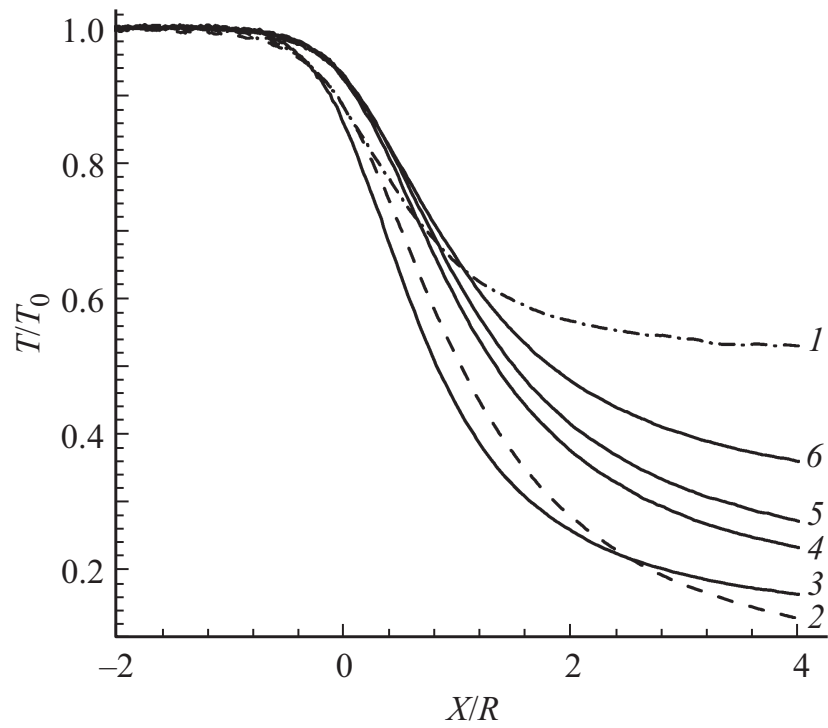

Рис. 2. Распределение температуры смеси вдоль оси течения. Номера кривых соответствуют номерам вариантов в таблице.

на бесконечном удалении от него $\left(n_{0,1}=X_{0,1} n_{0}-\right.$ плотность пара серебра внутри источника). Такое поведение плотности продемонстрировано на рис. $1, a$ (кривые $1-\mathrm{Kn}=2.83,2-\mathrm{Kn}=2.83 \cdot 10^{-3}, 3-$ истечение чистого пара серебра). При истечении бинарной смеси с большим отношением масс компонентов на зависимости безразмерной плотности тяжелого компонента (серебра) появляется максимум $n_{1} / n_{0,1}>1$, расположенный вблизи отверстия вниз по потоку (рис. 1, $a$, кривые 4-6). Величина данного максимума возрастает с увеличением мольной доли гелия в смеси и для варианта $X_{0,1}=0.05$ составляет $n_{1} / n_{0,1} \approx 1.5$.

Достоверность указанного поведения плотности наблюдалась авторами также при проведении дополнительных расчетов с использованием альтернативного программного кода [8] и иных вычислительных параметров (параметров расчетной сетки, числа частиц и пр.). Результаты расчетов совпали с представленными в пределах статистической погрешности и на рис. 1 не показаны.

Следует обратить внимание на то, что кривые безразмерной плотности серебра для вариантов 4-6 $\left(\mathrm{Kn} \approx 3 \cdot 10^{-2}, X_{0,1} \leqslant 0.25\right)$ лежат выше кривой, соответствующей варианту 2 для околоконтинуального течения $\left(\mathrm{Kn}=2.83 \cdot 10^{-3}, X_{0,1}=0.25\right)$. С уменьшением мольной доли серебра величина $n_{1} / n_{0,1}$ в струе возрастает.

Наличие эффекта роста плотности тяжелого компонента имеет важное значение. Во-первых, это важно с точки зрения возможного ускорения процесса формирования субнаноразмерных кластеров в истекающей струе. Частота тройных столкновений $\mathrm{Ag}-\mathrm{Ag}-\mathrm{Ag}$ зависит от плотности серебра как $n_{1}^{3}$, а частота столкновений $\mathrm{Ag}-\mathrm{Ag}-\mathrm{He}-$ как $n_{1}^{2}$. Частота тройных столкновений 

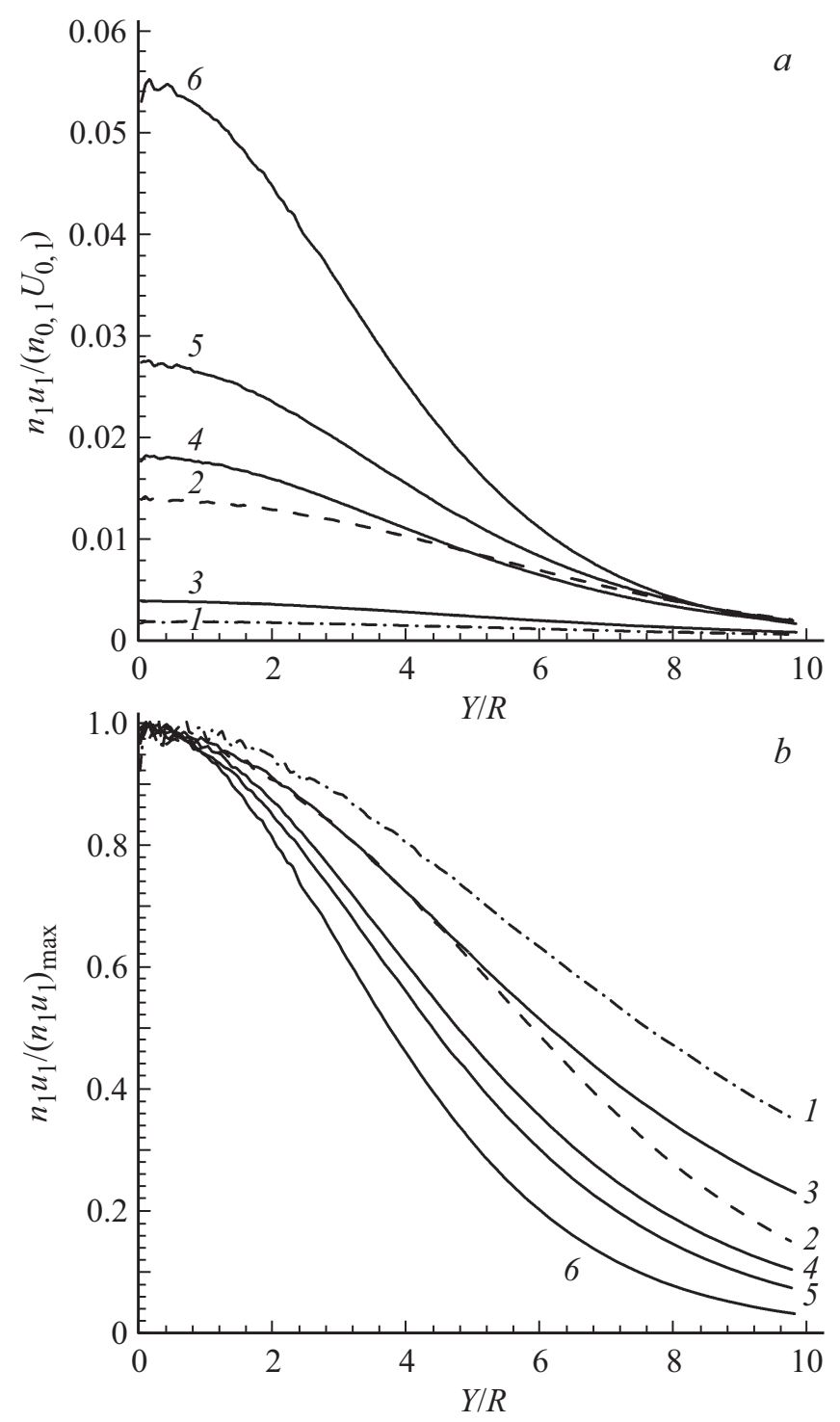

Рис. 3. Распределение потока $n_{1} u_{1}$ в плоскости $X / R=12 . a-$ $n_{1} u_{1} /\left(n_{0,1} U_{0,1}\right), b-n_{1} u_{1} /\left(n_{1} u_{1}\right)_{\max }$. Номера кривых соответствуют номерам вариантов в таблице.

наряду со средним значением относительных скоростей сталкивающихся частиц (зависящим от температуры) определяет скорость прямой реакции процесса образования димеров металла. Согласно данным рис. 2, температура разреженной смеси слабо отличается от температуры пара для случая расширения однокомпонентного газа (при той же парциальной плотности $n_{0,1}$ серебра внутри источника). Различие в плоскости выходного отверстия источника составляет примерно 7\%. Поэтому можно ожидать, что рост плотности приведет к интенсификации процесса формирования малых кластеров в непосредственной близости от отверстия. Изучение процессов кластеризации, имеющих важное значение для технологий синтеза кластеров в струе (cluster beam deposition) и технологий ВГСО, представляет собой отдельную задачу [9].
Во-вторых, увеличение плотности серебра скажется на росте расхода металла через выходное отверстие. Расход металла имеет принципиальное значение для технологий ВГСО, так как определяет скорость напыления.

Помимо плотности существенное влияние на рост расхода оказывает изменение скорости серебра в поле течения, в том числе в плоскости выходного отверстия. Изменение скорости вдоль оси приведено на рис. $1, b$. Течение смеси для варианта $1\left(\mathrm{Kn}=2.83, X_{0,1}=0.25\right)$ близко к бесстолкновительному. Замороженное значение скорости серебра в струе близко к средней тепловой скорости серебра в источнике $U_{0,1}=\left(8 k T_{0} /\left(\pi m_{1}\right)\right)^{1 / 2}$ (кривая 1). С уменьшением числа Кнудсена (ростом числа столкновений в струе) возрастает скорость паров серебра (кривая $4-\mathrm{Kn}=2.83 \cdot 10^{-2}, X_{0,1}=0.25$, кривая $\left.2-\mathrm{Kn}=2.83 \cdot 10^{-3}, X_{0,1}=0.25\right)$. Рост скорости серебра имеет место и при увеличении мольной доли гелия $X_{0,2}=1-X_{0,1}$ (кривые $4-6$ ). Этот эффект связан с ускорением атомов серебра легким гелием. Для гелия тепловая скорость в источнике и максимальная скорость стационарного расширения в вакуум отличаются от соответствующих значений для серебра в $\left(m_{1} / m_{2}\right)^{1 / 2}=5.19$ раза $(m-$ масса атома $)$.

Таким образом, одновременное влияние двух факторов (увеличение плотности и скорости тяжелого компонента) приводит к росту безразмерного потока атомов серебра через отверстие (рис. 1,c).

Рост расхода паров серебра также может быть продемонстрирован зависимостями $n_{1} u_{1}\left(u_{1}-\right.$ продольная компонента скорости атомов серебра) от поперечной координаты $Y$ в сечении $X / R=12$. Как следует, из данных, представленных на рис. $3, a$, в случае наличия гелия в смеси безразмерный расход серебра существенно возрастает по отношению к расходу, наблюдаемому как при свободномолекулярном или континуальном истечении, так и при истечении однокомпонентного газа. С ростом $X_{0,2}$ данный эффект усиливается. Рост мольной доли гелия приводит также к осевой фокусировке паров серебра (рис. $3, b)$.

Обнаруженные эффекты роста плотности паров тяжелого компонента смеси вблизи выходного отверстия источника, увеличения его расхода и осевой фокусировки позволят оптимизировать параметры газоструйных технологий синтеза кластеров и кластерных покрытий.

\section{Благодарности}

Расчеты выполнены с использованием ресурсов СКЦ „Политехнический“.

\section{Финансирование работы}

Работа выполнена при финансовой поддержке Министерства науки и высшего образования РФ. 


\section{Конфликт интересов}

Авторы заявляют, что у них нет конфликта интересов.

\section{Список литературы}

[1] Смирнов Б.М. // УФН. 2017. Т. 187. № 12. С. 1329-1364.

[2] Jamkhande P.G., Ghule N.W., Bamer A.H., Kalaskar M.G. // J. Drug Delivery Sci. Technol. 2019. V. 53. N 6. P. 101174.

[3] Быков Н.Ю., Сафонов А.Ю., Лещев Д.В., Старинский С.В., Булгаков А.В. // ЖТФ. 2019. Т. 89. В. 6. С. 830-843.

[4] Bird G.A. The DSMC method. Create Space Independent Publishing Platform, 2013. $300 \mathrm{p}$.

[5] Берд Г.А. Молекулярная газовая динамика. М.: Мир, 1980. $319 \mathrm{c}$.

[6] Химическая энциклопедия. Т. 4 / Под ред. Н.С. Зефирова. М.: Большая Российская энциклопедия, 1995. 639 с.

[7] Bykov N.Y., Gorbachev Yu.E. // Vacuum. 2019. V. 163. P. 119127.

[8] Zakharov V.V., Crifo J.-F., Rodionov A.V., Rubin M., Altwegg K. // Astronomy \& Astrophysics. 2018. V. 618. P. A71 $(1-16)$.

[9] Ivanov I.E., Nazarov V.S., Gidaspov V.Yu., Kryukov I.A. Numerical simulation of the process of phase transitions in gas-dynamic flows in nozzles and jets // Advances in theory and practice of computational mechanics. Smart innovation, systems and technologies. Springer Singapore, 2020. V. 173. P. 133-152. https://doi.org/10.1007/978-981-15-2600-8_11 